\title{
Development of an Improved Menopausal Symptom-Alleviating Licorice (Glycyrrhiza uralensis) by Biotransformation Using Monascus albidulus
}

\author{
Kang Uk Kim ${ }^{1}$, Sung-Jin Lee ${ }^{2}$, and Inhyung Lee ${ }^{1 *}$ \\ ${ }^{1}$ Department of Bio and Fermentation Convergence Technology, BK21 PLUS Project, Kookmin University, Seoul 02707, Republic of Korea \\ ${ }^{2}$ Food RED Center, SK Bioland Co., Ltd., Gyeonggi 15407, Republic of Korea
}

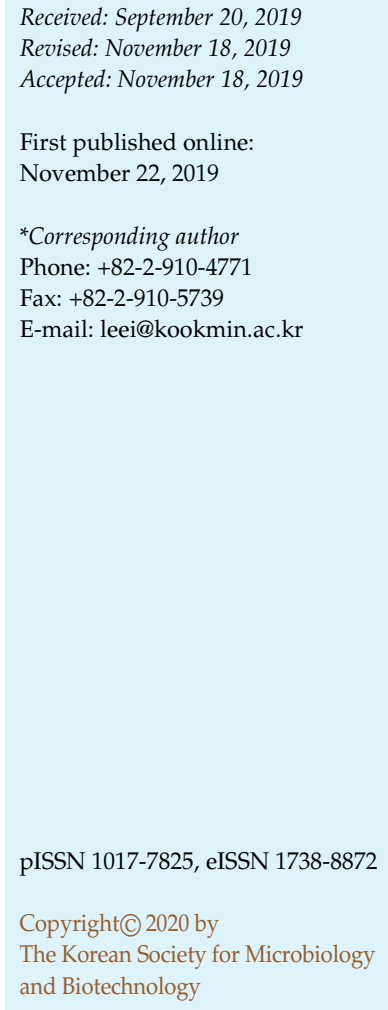

Licorice (Glycyrrhiza uralensis) contains several compounds that have been reported to alleviate menopausal symptoms via interacting with estrogen receptors (ERs). The compounds exist mainly in the form of glycosides, which exhibit low bioavailability and function. To bioconvert liquiritin and isoliquiritin, the major estrogenic compounds, to the corresponding deglycosylated liquiritigenin and isoliquiritigenin, respectively, licorice was fermented with Monascus, which has been demonstrated to deglycosylate other substances. The contents of liquiritigenin and isoliquiritigenin in Monascus-fermented licorice increased by 10.46-fold (from $38.03 \mu \mathrm{M}$ to $379.75 \mu \mathrm{M}$ ) and 12.50 -fold (from $5.53 \mu \mathrm{M}$ to $69.14 \mu \mathrm{M}$ ), respectively, compared with their contents in non-fermented licorice. Monascus-fermented licorice exhibited $82.5 \%$ of the ER $\beta$ binding activity of that observed in the positive control (17 $\beta$-estradiol), whereas the non-fermented licorice exhibited $54.1 \%$ of the binding activity in an in vivo ER binding assay. The increase in the ER $\beta$ binding activity was associated with increases in liquiritigenin and isoliquiritigenin contents. Liquiritigenin acts as a selective ligand for ER $\beta$, which alleviates menopausal symptoms with fewer side effects, such as heart disease and hypertension, compared with a ligand for ER $\alpha$. In addition, Monascus-fermented licorice contained $731 \mathrm{mg} / \mathrm{kg}$ of monacolin $\mathrm{K}$, one of the metabolites produced by Monascus that reduces serum cholesterol. Therefore, Monascus-fermented licorice is a promising material for the prevention and treatment of menopausal syndrome with fewer side effects.

Keywords: Monascus albidulus, licorice, menopausal symptoms, liquiritigenin, estrogen receptor $\beta$, monacolin $\mathrm{K}$

\section{Introduction}

Decreased levels of estrogens cause menopause syndrome in women after middle age. Menopausal symptoms, such as hot flushes, night sweats, depression, sleep disturbances, vaginal dryness, bone loss, and changes in cardiovascular and metabolic function, considerably affect the quality of life for middle-aged women [3,33]. Hormone replacement therapy (HRT) that uses pharmaceutical estrogens has been adopted to treat menopausal symptoms [10]. However, according to the Women's Health Initiative (WHI), HRT potentially has side effects that could lead to breast cancer and heart attacks, although it can effectively alleviate menopausal symptoms [17]. Therefore, HRT is limited to treatment of menopausal symptoms with minimal doses of replaced hormones [7]. Currently, many women prefer natural substitutes, such as herbal preparations or nutraceuticals, which are considered generally safer compared with HRT. Phytoestrogens are plant compounds that are considered to act similar to estrogens based on their analogous chemical structures [7]. Isoflavones, lignans, and coumestans are the major phytoestrogens [29]. Particularly, isoflavones, such as genistein and S-equol from soy, have been reported to be effective in alleviating 
menopausal symptoms $[1,15]$. However, the effectiveness of other phytoestrogens in the treatment of menopausal symptoms is yet to be evaluated. In addition, some reports have raised concerns over potential long-term side effects, such as stroke, heart disease, and breast cancer $[3,17,33]$. Therefore, currently, phytoestrogen has not been considered as a therapeutic agent.

Replaced hormones and phytoestrogens alleviate menopausal symptoms by binding to estrogen receptor alpha $(E R \alpha)$ and estrogen receptor beta (ER $\beta)[34,36]$. ER $\alpha$ and ER $\beta$ are very similar structurally; however, their ligand-binding domains are very different with regard to their selectivity to ligands [27]. ER $\alpha$ and ER $\beta$ exhibit entirely different functions [32]. Generally, when estrogenlike compounds bind selectively to ER $\beta$, they can function as tumor suppressors; therefore, estrogen-like compounds that bind to ER $\beta$ can alleviate menopausal symptoms without the side effects associated with menopausal therapies, such as breast cancer [32].

Licorice represents one of the most important traditional herbal medicines in Korea, China, Japan, and East Asia in general [44]. Some studies have suggested beneficial health effects of licorice and its bioactive constituents [18]. Licorice root extracts are often consumed as dietary supplements by women at menopause $[5,18]$. According to Boonmuen et al. (2016), licorice root extracts harbor diverse compounds, including ER agonists and antagonists [5, 18]. Particularly, liquiritigenin, isoliquiritigenin, calycosin, methoxychalcone, vestitol, and glycycoumarin are estrogen agonists. Among them, liquiritigenin, an aglycone form of liquiritin, is a well-known selective agonist for the tumor-suppressive ER $\beta$ [30]. Liquiritigenin is notably less stimulatory of genes promoting proliferation and motility when compared with $17 \beta$-estradiol (E2) [12]. Licorice is derived from three medicinal plants, Glycyrrhiza uralensis, Glycyrrhiza glabra, and Glycyrrhiza inflata. Each species has different compound profiles, and some compounds are specific markers for each species [40]. The contents of ER agonists, such as liquiritin, isoliquiritin, and liquiritigenin, are significantly higher in G. uralensis than in G. glabra or G. inflata. However, the mean content of liquiritigenin, an ER $\beta$ marker compound, in G. uralensis, is very low [21, 39]. Hwang et al. (2015) reported the bioconversion of liquiritin into liquiritigenin using Laetiporus sulphureus to increase liquiritigenin content in licorice; however, their study focused on liquiritin and liquiritigenin contents, but not on the bioconversion of associated compounds, such as liquiritin apioside and isoliquiritin [16].

Monascus spp. have been applied in the bioconversion of glycosides into their corresponding aglycones to enhance their functionality and bioavailability [14, 24]. In addition, Monascus spp. produce several bioactive compounds, such as monacolin $\mathrm{K}$ and $\gamma$-aminobutyric acid (GABA), during fermentation $[9,23,26]$, which could provide additional functionality to bioconversion effects. Particularly, monacolin $\mathrm{K}$ (also known as mevinolin or lovastatin) lowers blood cholesterol levels by inhibiting the activity of HMG-CoA reductase, a key enzyme in the cholesterol biosynthesis pathway [8]. Therefore, Monascus-fermented licorice could benefit women at menopause by enhancing cardiovascular functions.

In the present study, licorice was fermented with Monascus to enrich the preferred estrogen-like compounds that bind selectively to ER $\beta$. Several estrogenic compounds, including liquiritin and liquiritin apioside, were bioconverted into their corresponding aglycones, which could exhibit relatively higher functional activity and bioavailability. Monascusfermented licorice exhibited the high ER $\beta$ binding activity and contained monacolin K. Therefore, Monascus-fermented licorice is a promising and alternative functional material for the management of menopausal symptoms without the side effects associated with other common therapies.

\section{Materials and Methods}

\section{Chemicals and Media}

Monacolin K, citrinin, liquiritin, liquiritigenin, trifluoroacetic acid, acetic acid, and $p$-nitrophenyl- $\beta$-D-glucopyranoside were purchased from Sigma-Aldrich Co. (USA), isoliquiritin and isoliquiritigenin from ChemFaces (China), and liquiritin apioside and isoliquiritin apioside from Chengdu Biopurify Phytochemicals Ltd. (China). All HPLC-grade solvents were purchased from Fisher Scientific Korea Ltd. (Korea). Extraction solution and methanol were purchased from Samchun Pure Chemical (Korea). Potato dextrose agar (PDA) was purchased from Acumedia Inc. (USA), and glucose from Duksan Pure Chemicals (Korea). Yeast extract-peptone-dextrose and yeast nitrogen base media were purchased from Sigma-Aldrich Co. G. uralensis base media were composed of $2.0-5.0 \%$ G. uralensis extracts and $2 \%$ glucose. Licorice (G. uralensis) was purchased from a regional market in Chungcheongbuk-do, Korea. Licorice extracts were prepared by adding $5 \mathrm{~L}$ of distilled water to $1.2 \mathrm{~kg}$ of licorice, followed by boiling at $95^{\circ} \mathrm{C}$ for $5 \mathrm{~h}$. The extraction supernatant recovered by filtration with a Whatman filter paper No. 1 (UK) was concentrated to a solid content of $15 \%$ using a vacuum evaporator.

\section{Strains and Growth Conditions}

Thirteen Monascus strains that had been isolated from Monascus fermentation products were used [19, 20]. In addition, four type strains, Monascus pilosus KCCM 60084, Monascus purpureus KCCM 
Table 1. Contents of liquiritigenin and isoliquiritigenin, and monacolin K production in licorice fermented with Monascus strains.

\begin{tabular}{|c|c|c|c|c|c|c|c|}
\hline \multirow[b]{2}{*}{ Strain } & \multirow[b]{2}{*}{ Origin } & \multirow{2}{*}{$\begin{array}{c}\text { Fermented } \\
\text { solid contents } \\
(\%)\end{array}$} & \multicolumn{3}{|c|}{ Monacolin K (mg/kg) } & \multirow{2}{*}{$\begin{array}{l}\text { Liquiritigenin } \\
\text { (mg/g) }\end{array}$} & \multirow{2}{*}{$\begin{array}{l}\text { Isoliquiritigenin } \\
\qquad(\mathrm{mg} / \mathrm{g})\end{array}$} \\
\hline & & & Acid form & Lactone form & Total & & \\
\hline M. pilosus A & Red mold rice, Korea & $48.90 \pm 2.37$ & $834.06 \pm 176.20$ & $2,198.29 \pm 188.64$ & $3,032.35 \pm 247.99$ & $0.74 \pm 0.16$ & $0.39 \pm 0.07$ \\
\hline M. purpureus B & Red mold rice, Korea & $56.26 \pm 4.12$ & $136.86 \pm 27.48$ & $389.72 \pm 44.26$ & $526.58 \pm 57.89$ & $1.73 \pm 0.22$ & $0.22 \pm 0.06$ \\
\hline $\begin{array}{l}\text { M. pilosus } \\
\text { KMU106 }\end{array}$ & Red mold rice, Korea & $54.59 \pm 2.37$ & $142.40 \pm 37.41$ & $\mathrm{ND}^{c}$ & $142.4 \pm 37.41$ & $2.66 \pm 0.31$ & $0.42 \pm 0.08$ \\
\hline M. pilosus D & Red mold rice, Korea & $52.48 \pm 2.16$ & $142.79 \pm 11.16$ & ND & $142.79 \pm 11.16$ & $0.74 \pm 0.11$ & $0.31 \pm 0.12$ \\
\hline M. pilosus F & Red mold rice, Korea & $50.13 \pm 5.37$ & $139.28 \pm 18.14$ & ND & $139.28 \pm 18.14$ & $0.61 \pm 0.14$ & $0.13 \pm 0.04$ \\
\hline $\begin{array}{l}\text { M. pilosus } \\
\text { KMU108 }\end{array}$ & Red mold rice, Korea & $53.31 \pm 4.18$ & $147.45 \pm 25.18$ & ND & $147.45 \pm 25.18$ & $3.83 \pm 0.71$ & $0.60 \pm 0.04$ \\
\hline M. pilosus I & Red mold rice, Korea & $52.43 \pm 3.76$ & $146.46 \pm 31.43$ & ND & $146.46 \pm 31.43$ & $3.60 \pm 0.34$ & $0.63 \pm 0.05$ \\
\hline M. pilosus $\mathrm{K}$ & $\begin{array}{l}\text { Red mold rice powder, } \\
\text { China }\end{array}$ & $45.96 \pm 3.72$ & $807.37 \pm 90.11$ & $2,426.45 \pm 213.86$ & $3,233.82 \pm 300.41$ & $1.87 \pm 0.18$ & $0.34 \pm 0.05$ \\
\hline M. pilosus M & $\begin{array}{l}\text { Red mold rice powder, } \\
\text { China }\end{array}$ & $52.19 \pm 6.54$ & $151.09 \pm 21.87$ & ND & $151.09 \pm 21.87$ & $3.18 \pm 0.26$ & $0.53 \pm 0.12$ \\
\hline M. albidulus $\mathrm{N}$ & $\begin{array}{l}\text { Red mold rice powder, } \\
\text { China }\end{array}$ & $44.82 \pm 3.11$ & $874.01 \pm 48.64$ & $2,870.10 \pm 128.19$ & $3,744.11 \pm 166.87$ & $3.22 \pm 0.31$ & $0.40 \pm 0.08$ \\
\hline M. pilosus O & $\begin{array}{l}\text { Red mold rice powder, } \\
\text { China }\end{array}$ & $52.67 \pm 1.48$ & $130.90 \pm 12.89$ & ND & $130.9 \pm 12.89$ & $3.14 \pm 0.48$ & $0.64 \pm 0.08$ \\
\hline M. pilosus T & $\begin{array}{l}\text { Red mold rice powder, } \\
\text { China }\end{array}$ & $54.22 \pm 2.86$ & $130.90 \pm 6.48$ & ND & $130.9 \pm 6.48$ & $2.24 \pm 0.29$ & $0.67 \pm 0.11$ \\
\hline M. pilosus U & $\begin{array}{l}\text { Red mold rice powder, } \\
\text { China }\end{array}$ & $51.68 \pm 5.12$ & $140.58 \pm 17.78$ & ND & $140.58 \pm 17.78$ & $3.94 \pm 0.34$ & $0.68 \pm 0.16$ \\
\hline $\begin{array}{l}\text { M. pilosus } \\
\text { KCCM60084 }\end{array}$ & $\mathrm{KCCM}^{\mathrm{a}}$ & $53.33 \pm 3.42$ & $142.64 \pm 22.68$ & ND & $142.64 \pm 22.68$ & $3.44 \pm 0.16$ & $0.63 \pm 0.07$ \\
\hline $\begin{array}{l}\text { M. purpureus } \\
\text { KCCM60016 }\end{array}$ & KCCM & $55.30 \pm 3.76$ & $144.77 \pm 17.64$ & $39.18 \pm 2.84$ & $183.95 \pm 20.68$ & $2.19 \pm 0.15$ & $0.38 \pm 0.08$ \\
\hline $\begin{array}{l}\text { M. ruber } \\
\text { KCTC6122 }\end{array}$ & $\mathrm{KCTC}^{\mathrm{b}}$ & $57.38 \pm 4.29$ & $145.13 \pm 7.66$ & $225.99 \pm 29.87$ & $371.12 \pm 38.04$ & $0.37 \pm 0.08$ & $0.16 \pm 0.08$ \\
\hline $\begin{array}{l}\text { M. kaoliang } \\
\text { KCCM60154 }\end{array}$ & КССM & $53.13 \pm 4.86$ & $152.62 \pm 10.04$ & $92.59 \pm 11.64$ & $245.21 \pm 20.80$ & $3.18 \pm 0.24$ & $0.52 \pm 0.12$ \\
\hline
\end{tabular}

${ }^{a}$ KCCM, Korea Culture Center of Microorganisms.

${ }^{\mathrm{b}}$ KCTC, Korean Collection for Type Cultures.

${ }^{\mathrm{c}} \mathrm{ND}$, not detected.

Fermentation was carried out at $30^{\circ} \mathrm{C}$ with shaking at $200 \mathrm{rpm}$ for $150 \mathrm{~h}$ in a $2 \%$ licorice medium.

60016, Monascus ruber KCTC 6122, and Monascus kaoliang KCCM 60154, obtained from the Korea Culture Center of Microorganisms (KCCM, Korea) or the Korean Collection for Type Cultures (KCTC, Korea), were used in the initial screening (Table 1).

Spores were harvested using a $0.85 \%$ saline buffer after culturing each of the strains on PDA plates for 5 days at $30^{\circ} \mathrm{C}$. Spores were inoculated into $50 \mathrm{ml}$ of $\mathrm{G}$. uralensis base media at $1.0 \times 10^{6} \mathrm{spore} / \mathrm{ml}$. A $2 \%$ or $5 \%$ licorice medium was used for the initial screening or licorice fermentation. All broth cultures were incubated for 3 days (initial screening) or 10 days (fermentation) at $30^{\circ} \mathrm{C}$ with constant shaking at $200 \mathrm{rpm}$. The culture broth was freeze-dried using a freeze-dryer (FDU-1200, EYELA, Japan) for the analysis of fermentation products.

For an in vivo ER binding assay, the yeasts harboring pRR-ERß5Z (plasmid \#23062) or pRR-ER $\alpha-5 Z$ (plasmid \#23061) were constructed by transforming each plasmid into Saccharomyces cerevisiae W300a obtained from Prof. S. J. Park (Yonsei University, Seoul, Korea) [11, 31]. Both plasmids were purchased from Addgene (USA). Yeast transformation was carried out using an MP BIO Alkali-Cation Yeast Transformation Kit (MP Biomedicals, USA) according to the kit protocol. Yeast was routinely cultured and maintained on a minimal media [11]. 
Quantitative Analysis of Monacolin K, Citrinin, Liquiritin, Liquiritin Apioside, Liquiritigenin, Isoliquiritin, Isoliquiritin Apioside, and Isoliquiritigenin

Quantitative analyses of monacolin $\mathrm{K}$ and citrinin were carried out using HPLC as described previously for the analysis of Monascus-fermented red ginseng [14]. Liquiritin, isoliquiritin, liquiritigenin, isoliquiritigenin, liquiritin apioside and isoliquiritin apioside were also analyzed quantitatively [21] using an Agilent HPLC equipped with a HiQ sil C18W column $(250 \times 4.6 \mathrm{~mm}$, particle size $5 \mu \mathrm{m}$, pore size $120 \AA$; Kya Tech Corporation, Japan). For each chemical analysis, $50 \mathrm{mg}$ of a sample was extracted using $500 \mu \mathrm{l}$ of methyl alcohol for $40 \mathrm{~min}$ with gentle shaking. All extracted samples were filtered using a $0.45-\mu l$ syringe filter (Life Sciences, USA). For the liquiritin, liquiritin apioside, isoliquiritin and isoliquiritn apioside HPLC analyses, samples $(20 \mu \mathrm{l})$ were separated using an acetonitrile gradient mobile phase $(20 \%$ acetonitrile for $10 \mathrm{~min}$ for the initial run, followed by $20-40 \%$ gradient for $2 \mathrm{~min}, 40 \%$ for $13 \mathrm{~min}, 40-20 \%$ gradient for $2 \mathrm{~min}$, and $20 \%$ for $23 \mathrm{~min}$ ) at a flow rate of $0.5 \mathrm{ml} / \mathrm{min}$. Signals were detected using a UV detector (Agilent 1260 Infinity LC, Agilent Technologies, USA) at a wavelength of 254 and $370 \mathrm{~nm}$ for liquiritin and liquiritin apioside and for isoliquiritin and isoliquiritin apioside, respectively. For the liquiritigenin and isoliquiritigenin HPLC analyses, samples $(20 \mu \mathrm{l})$ were separated using an acetonitrile gradient mobile phase (10-30\% acetonitrile gradient for $10 \mathrm{~min}$ for the initial run, followed by $30-50 \%$ gradient for $35 \mathrm{~min}, 50-70 \%$ gradient for $10 \mathrm{~min}, 70-75 \%$ gradient for $10 \mathrm{~min}, 75-10 \%$ for $10 \mathrm{~min}$, and $10 \%$ for $10 \mathrm{~min}$ ) at a flow rate of $1 \mathrm{ml} / \mathrm{min}$. Signals were detected at a wavelength of 274-nm and 370-nm wavelengths for liquiritigenin and for isoliquiritigenin, respectively.

\section{$\beta$-Glucosidase Assay}

$\beta$-Glucosidase in the culture broth was measured as described previously with minor modifications [14]. Substrate solution was prepared by dissolving $p$-nitrophenyl- $\beta$-D-glucopyranoside in a $50 \mathrm{mM}$ potassium phosphate buffer. Five hundred microliters of the aliquot was stored at $-20^{\circ} \mathrm{C}$ until use. Two hundred microliters of supernatant from the culture broths was centrifuged at $13,750 \times g$ for $10 \mathrm{~min}$, mixed with $500 \mu \mathrm{l}$ of preheated substrate solution, and then incubated at $30^{\circ} \mathrm{C}$ for $30 \mathrm{~min}$. Released $p$ nitrophenol was measured using a spectrophotometer at $405 \mathrm{~nm}$. One unit of $\beta$-glucosidase activity was defined as the release of 1 $\mu$ mole of $p$-nitrophenol per min.

\section{Yeast Estrogen Binding Assay}

The ER $\alpha$ and $\beta$ binding assays were performed using $S$. cerevisiae harboring pRR-ER $\alpha-5 Z$ or pRR-ER $\beta-5 Z$ [31] as described by Fox (2008) [11]. Yeast was pre-cultured in a minimal medium for approximately $24 \mathrm{~h}$ until the optical density (OD) at $600 \mathrm{~nm}$ exceeded 1. Subsequently, culture was diluted to OD $=0.4$ with a fresh medium. Diluted yeast culture $(200 \mu \mathrm{l})$ and $5 \mu \mathrm{l}$ of Monascusfermented licorice extract were transferred to a 96 -well plate. The plate was incubated for $18 \mathrm{~h}$ at $30^{\circ} \mathrm{C}$. Culture $(50 \mu \mathrm{l})$ was mixed with a $200 \mu \mathrm{l}$ of Lac-Z buffer in a new 96-well plate, and then incubated at $30^{\circ} \mathrm{C}$ for $15 \mathrm{~min}$. Color development was monitored at $405 \mathrm{~nm}$. The ER binding activity was expressed as \% relative absorbance to the $17 \beta$-estradiol treatment whose maximum absorbance was set to $100 \%$ [11].

\section{Results and Discussion}

Screening for Monascus Strains Suitable for the Bioconversion of Liquiritin and Isoliquiritin in Licorice (G. uralensis)

We have reported previously that Monascus spp. can convert glycosides into their corresponding aglycones [14, 24]. In the present study, we attempted to bioconvert the glycosidic components in licorice, such as liquiritin and liquiritin apioside, into liquiritigenin, a well-known selective agonist for ER $\beta$. First, we tested and screened for Monascus strains suitable for the deglycosylation of liquiritin and isoliquiritin in licorice. We have previously isolated 17 Monascus strains from Monascus-fermented products [19, 20]. Each strain exhibited the strain-specific characteristics with regard to enzyme and secondary metabolite production, such as monacolin K, pigments, and citrinin, under different fermentation substrates [14, 24]. Therefore, the strains were evaluated for the deglycosylation of liquiritin and isoliquiritin and for the production of monacolin $\mathrm{K}$ in licorice media. Liquiritin and isoliquiritin in licorice were all converted into the corresponding deglycosylated compounds by fermentation with Monascus spp. The liquiritin peak (retention time $17.2 \mathrm{~min}$ ) disappeared (Fig. 1A), and the liquiritigenin peak (retention time $15.8 \mathrm{~min}$ ) emerged (Fig. 1B) after 3 days of fermentation. Similarly, the isoliquiritin peak (retention time $20.9 \mathrm{~min}$ ) disappeared (Fig. 1C) and the isoliquiritigenin peak (retention time $24.8 \mathrm{~min}$ ) was observed (Fig. 1D) after 3 days of fermentation.

The liquiritigenin and isoliquiritigenin contents in the freeze-dried Monascus-fermented licorice ranged from 0.37 to $3.94 \mathrm{mg} / \mathrm{g}$ and from 0.13 to $0.68 \mathrm{mg} / \mathrm{g}$, respectively (Table 1). M. pilosus $\mathrm{U}$ exhibited the highest bioconversion activities for liquiritigenin and isoliquiritigenin, which were observed at 3.94 and $0.68 \mathrm{mg} / \mathrm{g}$, respectively, and was followed by M. pilosus KMU108, M. pilosus I, and M. albidulus $\mathrm{N}$ (Table 1). On the other hand, most strains produced monacolin $\mathrm{K}$ at various levels, ranging from 130.9 to $3,744.1 \mathrm{mg} / \mathrm{g}$, and M. pilosus A, M. purpureus B, $M$. pilosus $\mathrm{K}$, and M. albidulus $\mathrm{N}$ produced over $500 \mathrm{mg} / \mathrm{g}$ of monacolin $\mathrm{K}$, which is the minimum level to be claimed as a functional food in the case of Monascus-fermented red 
A

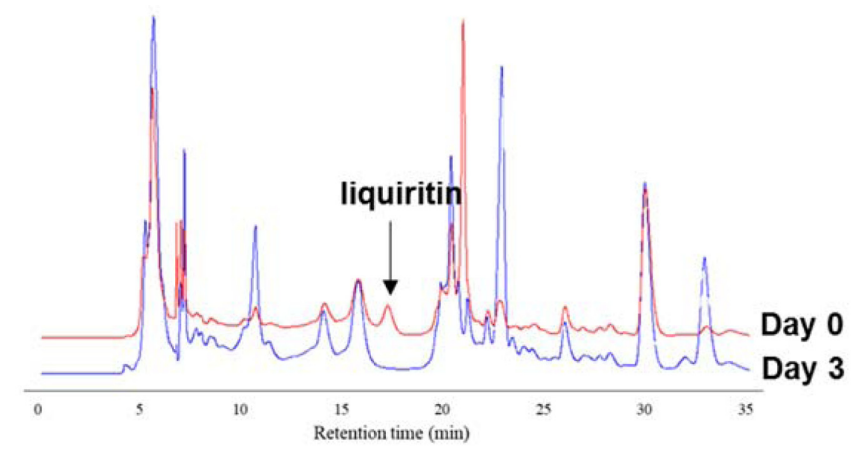

C

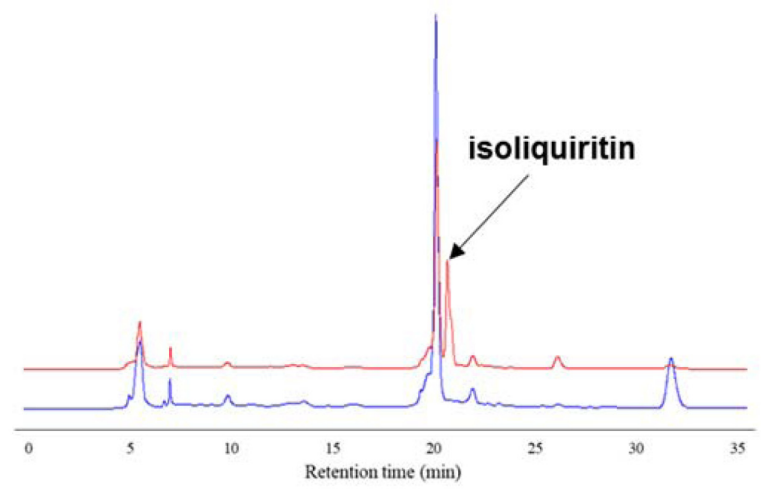

B

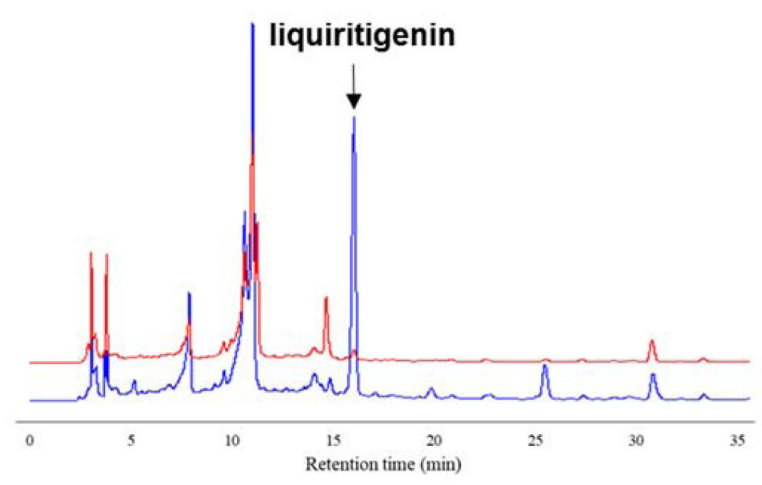

D

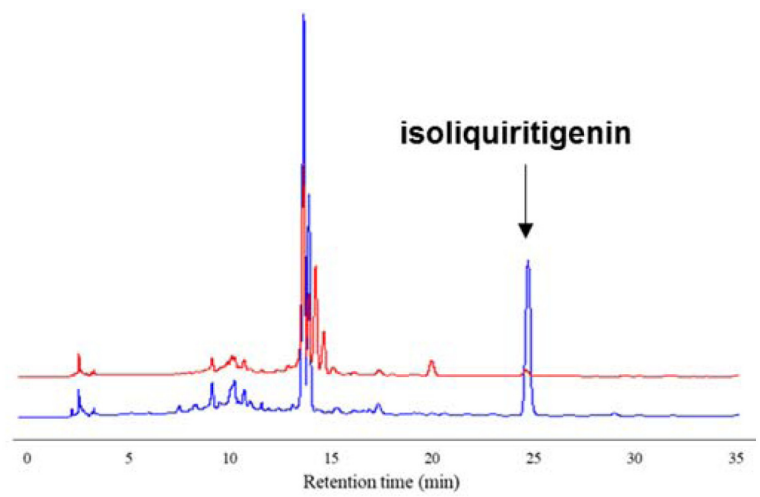

Fig. 1. HPLC chromatograms of liquiritin (A), liquiritigenin (B), isoliquiritin (C), and isoliquiritigenin (D).

yeast rice (2014 Health Functional Food Code, Ministry of Food and Drug Safety (MFDS), Korea). In addition, most of the strains produced citrinin at very low concentrations, and citrinin was not detected in M. albidulus N-fermented licorice (data not shown). Since citrinin is a nephrotoxic and hepatotoxic fungal toxin $[4,22]$, the selection of a strain that produces little or no citrinin is very critical for functional food materials. The level of citrinin production in Monascus spp. is known to be substrate dependent [35]. Therefore, M. albidulus $\mathrm{N}$ was selected as the strain suitable for the bioconversion of liquiritin and isoliquiritin in licorice. It has been identified previously as M. albidulus $[19,20]$ and was designated as M. albidulus KMU112.

\section{Bioconversion of Liquiritin and Isoliquiritin in Licorice during Fermentation with M. albidulus KMU112}

The liquiritin concentration was $149.64 \mu \mathrm{M}$, whereas that of liquiritigenin was very low at $38.03 \mu \mathrm{M}$ in a $5 \%$ preculture licorice medium (Fig. 2A), suggesting that most of liquiritin exists in a glycosylated form in licorice. After 3 days of fermentation, liquiritin was no longer detected, and liquiritigenin concentrations increased to $322.63 \mu \mathrm{M}$ on day
4 of fermentation and reached a maximum of $397.75 \mu \mathrm{M}$ on day 9 of fermentation (Fig. 2A). Such an unexpected increase in the concentration of liquiritigenin suggested the existence of liquiritin analogs in licorice and their bioconversion into liquiritigenin during Monascus fermentation. Liquiritin apioside is another liquiritin glycoside (Fig. 3), observed at $1.27 \%$ on average in G. uralensis and more than $1 \%$ in various licorice species [21]. Consequently, some of liquiritigenin could have been derived from liquiritin apioside, and the concentrations were $712.05 \mu \mathrm{M}$ before fermentation and $383.82 \mu \mathrm{M}$ on day 3 of fermentation, respectively (Fig. 2A).

The concentrations of isoliquiritin and isoliquiritigenin were $40.99 \mu \mathrm{M}$ and $5.53 \mu \mathrm{M}$, respectively, in the licorice medium, suggesting that a glycolylated form has higher concentration than an aglycoylated form, such as liquiritin/ liquiritgenin. Isoliquiritin was also not detected after 3 days of fermentation, and the concentrations of isoliquiritigenin increased steadily during the fermentation period and reached a maximum of $69.14 \mu \mathrm{M}$ on day 9. Some of the isoliquiritigenin could also be derived from isoliquiritin apioside, which is an isoliquiritin glycoside (Fig. 3). Such bioconversion was supported by the $30.63 \%$ reduction in 

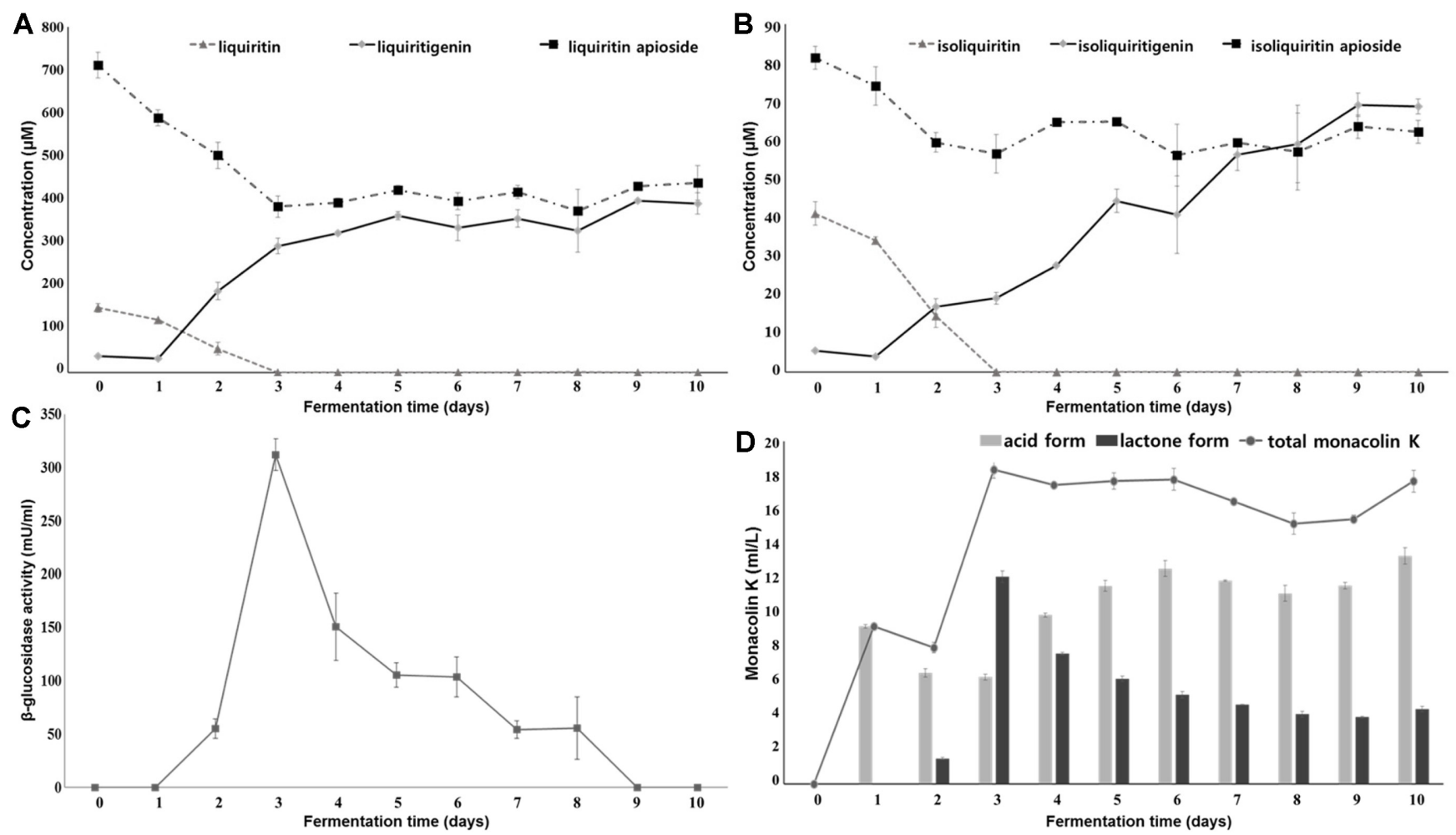

Fig. 2. Bioconversion of liquiritin/liquiritin apioside and isoliquiritin/isoliquiritin apioside, $\beta$-glucosidase activity, and monacolin K production during licorice fermentation.

(A) Bioconversion of liquiritin and liquiritin apioside to liquiritigenin; (B) bioconversion of isoliquiritin and isoliquiritin apioside to isoliquiritigenin; (C) $\beta$-glucosidase activity; (D) monacolin K production.

A

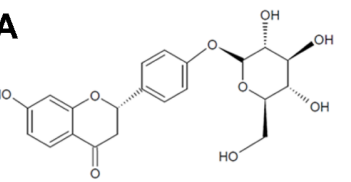

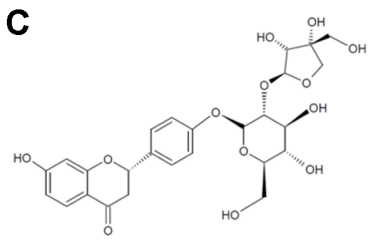

D

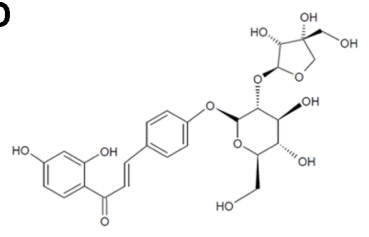

$\mathbf{F}$

E<smiles>CC(C)COc1ccc(C(=O)/C=C/c2ccc(O)cc2)c(O)c1</smiles>

Fig. 3. Chemical structures of liquiritin (A), isoliquiritin (B), liquiritin apioside (C), isoliquiritin apioside (D), liquiritigenin $(\mathrm{E})$, and isoliquiritigenin $(\mathbf{F})$. isoliquiritin apioside from $81.32 \mu \mathrm{M}$ to $56.41 \mu \mathrm{M}$ on the day 3 culture (Fig. 2B).

Liquiritin and isoliquiritin are bioconverted into liquiritigenin and isoliquiritigenin by $\beta$-glucosidase $[2,45]$. The $\beta$-glucosidase activity was detected in fermented licorice and reached a maximum of $312.02 \mathrm{mU} / \mathrm{ml}$ after 3 days of fermentation, suggesting that liquiritin and isoliquiritin of licorice were bioconverted by $\beta$-glucosidase in M. albidulus KMU112 (Fig. 2C). Conversely, the concentrations of liquiritin apioside and isoliquiritin apioside decreased until day 3 and then maintained a constant concentration, and the decreases represented $46.10 \%$ and $30.63 \%$ reductions from the initial concentrations, respectively. The reason for the partial bioconversion of liquiritin/isoliquiritin apiosides remains unclear; however, apiosides seem to be converted in different ways compared with liquiritin/isoliquiritin. According to Sato (2007) [37], liquiritin/liquiritin apioside and isoliquiritin/isoliquiritin apioside were bioconverted completely by naringinase, which has both $\alpha$-L-rhamnosidase and $\beta$-glucosidase functions. Therefore, liquiritin apioside and isoliquiritin apioside could be bioconverted by 
enzymes other than $\beta$-glucosidase [37].

Since liquiritigenin and isoliquiritigenin can be interconverted naturally [38], the final liquiritigenin and isoliquiritigenin concentrations may not be proportional to each bioconversion. In summary, glycosylated forms of liquiritin/isoliquiritin and liquiritin apioside/isoliquiritin apioside were successfully biotransformed into their corresponding deglycosylated forms by fermentation.

\section{Monacolin K Production during Licorice Fermentation by M. albidulus KMU112}

Total monacolin $\mathrm{K}$ was detected at $18.35 \mathrm{mg} / \mathrm{L}$ on day 3 of culture, and the concentration was maintained until day 10 of fermentation (Fig. 2D). The active monacolin K (acid form) was produced mainly during the early fermentation period (9.21 mg/l on day 1$)$, and then the concentration increased gradually up to the maximum level on day 6 of fermentation $(12.58 \mathrm{mg} / \mathrm{l})$. Conversely, the inactive monacolin K (lactone form) was detected after day 2, and reached a maximum $(12.11 \mathrm{mg} / \mathrm{l})$ on day 3 , followed by a gradual decrease. The $\mathrm{pH}$ of the culture broth decreased until day 3 of fermentation and then increased after, suggesting that an increase in the active acid form after day 3 was in part derived from the inactive lactone form due to change in $\mathrm{pH}$ [28]. The active and inactive monacolin $\mathrm{K}$ concentrations in the freeze-dried powder of the 10-day culture were $551.03 \mathrm{mg} / \mathrm{kg}$ and $180.55 \mathrm{mg} / \mathrm{kg}$, respectively. Monacolin $\mathrm{K}$ production has been demonstrated to be affected by substrates. The monacolin $\mathrm{K}$ concentrations in Monascus-fermented soybean, fortified mugwort, or ginseng were 1,366, 2,219, and 3,089 mg/kg [14, 24, 25]. In addition, the concentration of a substrate influences monacolin $\mathrm{K}$ production so that monacolin $\mathrm{K}$ yield was generally higher under lower substrate concentrations [41]. In the present study, $5 \%$ licorice medium instead of $2 \%$ licorice medium was used for strain screening to increase liquiritigenin and isoliquiritingenin concentrations. The amounts of active monacolin $\mathrm{K}$ in fermented licorice exceed the monacolin $\mathrm{K}$ standard concentrations in the Monascus-fermented red yeast rice functional food (2014 Health Functional Food Code, MFDS, Korea). Therefore, the fermented licorice could prevent the cardiovascular diseases associated with the adverse side effects of the female climacteric syndrome, which is an additional benefit of fermented licorice.

\section{In Vivo ER Binding Assay of Monascus-Fermented Licorice Extracts}

The ER $\alpha$ and ER $\beta$ binding activities of the Monascusfermented licorice extracts were evaluated using recombinant yeasts that contained the ER $\alpha$ or ER $\beta$ gene fused to a $\beta$ galactosidase reporter gene. The ER $\alpha$ and ER $\beta$ binding activities of the extracts of pre-fermented licorice media were $3.04 \%$ and $54.06 \%$ that of the positive control, $17 \beta$ estradiol, respectively (Fig. 4), which is consistent with the findings of a study where licorice exhibited higher ER $\beta$ binding activity compared with ER $\alpha$ binding activity [42]. Both ER $\alpha$ and ER $\beta$ binding activities increased by fermentation with $M$. albidulus. On day 4 of fermentation, the ER $\alpha$ binding activity reached to the maximum level, which was $22.0 \%$ that of the positive control. Conversely, the ER $\beta$ binding activity increased up to $82.54 \%$ with the extracts of the 8-day fermented licorice. However, the increase in ER binding activity was not proportional to the liquiritigenin and isoliquiritigenin concentrations, suggesting the involvement of other components in ER binding. Licorice contains not only estrogenic agonists but also estrogenic antagonists [5].

The ER $\alpha$ and ER $\beta$ binding activity of licorice has been reported using the MCF-7 cells; however, the binding activity was reported to be low compared with the positive control $[5,30]$. In the present study, we demonstrate that fermentation with Monascus could considerably increase the ER $\alpha$ and ER $\beta$ binding activities of licorice. Particularly, the ER $\beta$ binding activity was almost equivalent to that of the positive control. Such an increase in the selective binding capacity of fermented licorice to ER $\beta$ could be due to increased liquiritigenin concentrations in the course of fermentation. Liquiritigenin binds selectively to ER $\beta$ [30].

According to van Patten (2002), a very high binding activity for ER $\alpha$ is associated with phytoestrogen-induced side effects, such as breast cancer [43]. The most commonly

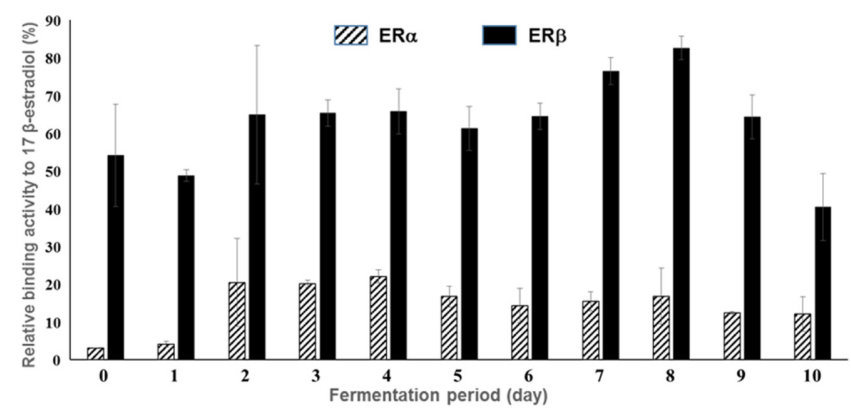

Fig. 4. ER binding activities of the licorice fermentation extracts.

The ER $\alpha$ and ER $\beta$-binding activities of licorice extracts were evaluated before and after fermentation using an in vivo assay system. ER binding activity was expressed as \% relative absorbance to the $17 \beta$ estradiol treatment whose maximum absorbance was set to $100 \%$. 
used phytoestrogens, soybean isoflavones, have a high binding affinity for ER $\alpha$. For example, genistein exhibits approximately $60 \% \mathrm{ER} \alpha$ binding ability [6]. In recent years, the Japanese Food Safety Commission has limited the daily intake of isoflavone to less than $30 \mathrm{mg} / \mathrm{kg}$ in a specialized diet, which was also adopted by MFDS in Korea [1, 13, 15]. The fermented licorice in the present study exhibited an ER binding activity, preferentially to ER $\beta$, of up to $82.5 \%$. Therefore, fermented licorice could have an advantage over other phytoestrogens in the alleviation of menopause symptoms with low side effects.

Market demand for women's menopausal remedies is on the rise due to growing interest in women's health. In the present study, Monascus-fermented licorice contained high levels of liquiritigenin and monacolin $\mathrm{K}$ and exhibited high ER $\beta$-selective binding activity. Therefore, Monascusfermented licorice is a promising material that could be applied in the prevention and treatment of menopausal syndrome with or without minimal side effects.

\section{Acknowledgments}

This work was supported by a grant from the National Research Foundation of Korea (No. 2017R1A2B4004748). D. H. Kim and D. Jeong are acknowledged for technical assistance.

\section{Conflict of Interest}

The authors have no financial conflicts of interest to declare.

\section{References}

1. Allred CD, Allred KF, Ju YH, Virant SM, Helferich WG. 2001. Soy diets containing varying amounts of genistein stimulate growth of estrogen-dependent (MCF-7) tumors in a dose-dependent manner. Cancer Res. 61: 5045-5050.

2. Aura A-M. 2008. Microbial metabolism of dietary phenolic compounds in the colon. Phytochem. Rev. 7: 407-429.

3. Avis NE, Stellato R, Crawford S, Bromberger J, Ganz P, Cain V, et al. 2001. Is there a menopausal syndrome? Menopausal status and symptoms across racial/ethnic groups. Soc. Sci. Med. 52: 345-356.

4. Blanc PJ, Laussac J, Le Bars J, Le Bars P, Loret M, Pareilleux A, et al. 1995. Characterization of monascidin A from Monascus as citrinin. Int. J. Food Microbiol. 27: 201-213.

5. Boonmuen N, Gong P, Ali Z, Chittiboyina AG, Khan I, Doerge DR, et al. 2016. Licorice root components in dietary supplements are selective estrogen receptor modulators with a spectrum of estrogenic and anti-estrogenic activities. Steroids 105: 42-49.

6. De Boever P, Demaré W, Vanderperren E, Cooreman K, Bossier P, Verstraete W. 2001. Optimization of a yeast estrogen screen and its applicability to study the release of estrogenic isoflavones from a soygerm powder. Environ. Health Perspect. 109: 691-697.

7. Depypere HT, Comhaire FH. 2014. Herbal preparations for the menopause: beyond isoflavones and black cohosh. Maturitas 77: 191-194.

8. Endo A. 1979. Monacolin K, a new hypocholesteroemic agent produced by a Monascus species. J. Antibiot. (Tokyo). 32: $852-854$.

9. Erdogrul L. 2004. Review of the studies on the red yeast rice (Monascus purpureus). Turkish Electro. J. Biotechnol. 2: 37-49.

10. Ettinger B. 1998. Overview of estrogen replacement therapy: a historical perspective. Proc. Soc. Exp. Biol. Med. 217: 2-5.

11. Fox JE, Burow ME, McLachlan JA, Miller III CA. 2008. Detecting ligands and dissecting nuclear receptor-signaling pathways using recombinant strains of the yeast Saccharomyces cerevisiae. Nat. Protoc. 3: 637-645.

12. Gong P, Madak-Erdogan Z, Li J, Cheng J, Greenlief CM, Helferich W, et al. 2014. Transcriptomic analysis identifies gene networks regulated by estrogen receptor $\alpha(E R \alpha)$ and ER $\beta$ that control distinct effects of different botanical estrogens. Nucl. Recept. Signal. 12: e001.

13. Hankinson SE, Eliassen AH. 2007. Endogenous estrogen, testosterone and progesterone levels in relation to breast cancer risk. J. Steroid Biochem. Mol. Biol. 106: 24-30.

14. Hong S-Y, Oh J-H, Lee I. 2011. Simultaneous enrichment of deglycosylated ginsenosides and monacolin $\mathrm{K}$ in red ginseng by fermentation with Monascus pilosus. Biosci. Biotechnol. Biochem. 75: 1490-1495.

15. Hsieh C-Y, Santell RC, Haslam SZ, Helferich WG. 1998. Estrogenic effects of genistein on the growth of estrogen receptor-positive human breast cancer (MCF-7) cells in vitro and in vivo. Cancer Res. 58: 3833-3838.

16. Hwang HJ, Jeong SC, Park JP. 2015. Production of liquiritigenin with cell-based biotrasformation and its antiaging activity. Kor. Soc. Biotechnol. Bioeng. J. 30: 166-174.

17. Writing Groups for the Women's Health Initiative Investigators. 2002. Risks and benefits of estrogen plus progestin in healthy postmenopausal women: principal results from the Women's Health Initiative randomized controlled trial. JAMA 288: 321-333.

18. Kao T-C, Wu C-H, Yen G-C. 2014. Bioactivity and potential helth benefits of licorice. J. Agric. Food Chem. 62: 542-553.

19. Kim H-J, Ji GE, Lee I. 2007. Natural occuring levels of citrinin and monacolin $\mathrm{K}$ in Korean Monascus fermentation products. Food Sci. Biotechnol. 16: 142-145.

20. Kim JY, Kim H-J, Oh J-H, Lee I. 2010. Characteristics of Monascus sp. isolated from Monascus fermentation products. Food Sci. Biotechnol. 19: 1151-1157. 
21. Kondo K, Shiba M, Nakamura R, Morota T, Shoyama Y. 2007. Constituent properties of licorices derived from Glycyrrhiza uralensis, G. glabra, or G. inflata identified by genetic information. Biol. Pharm. Bull. 30: 1271-1277.

22. Krejci ME, Bretz NS, Koechel DA. 1996. Citrinin produces acute adverse changes in renal function and ultrastructure in pentobarbital-anesthetized dogs without concomitant reductions in [potassium] plasma. Toxicology 106: 167-177.

23. Kuba-Miyara M, Yasuda M. 2012. Bioorganic compounds produced by the fungus Monascus and their use in health sciences ans medicine. Mini-Rev. Org. Chem. 9: 11-19.

24. Lee DS, Lee I. 2012. Development of monacolin K-enriched ganghwayakssuk (Artemisia princeps Pamp.) by fermentation with Monascus pilosus. J. Microbiol. Biotechnol. 22: 975-980.

25. Lim JY, Kim JJ, Lee DS, Kim GH, Shim JY, Lee I, Imm JY. 2010. Physicochemical characteristics and production of whole soymilk from Monascus fermented soybeans. Food Chem. 120: 255-260.

26. Lin YL. 2008. Biologically active components and nutraceuticals in the Monascus-fermented rice: a review. Appl. Microbiol. Biotechnol. 77: 965-973.

27. Lo R, Matthews J. 2010. A new class of estrogen receptor beta-selective activators. Mol. Interv. 10: 133-136.

28. Ma J, Li Y, Ye Q, Li J, Hua Y, Ju D, et al. 2000. Constituents of red yeast rice, a traditional Chinese food and medicine. J. Agric. Food Chem. 48: 5220-5225.

29. Mazur W, Adlercreutz H. 2000. Overview of naturally occuringendocrine-active substances in the human diet in relation to human health. Nutrition 16: 654-687.

30. Mersereau JE, Levy N, Staub RE, Baggett LF, Leitman DC. 2008. Liquiritigenin is a plant-derived highly selective estrogen receptor beta agonist. Mol. Cell. Endocrinol. 283: 49-57.

31. Miller III CA, Tan X, Wilson M, Bhattacharyya S, Ludwig S. 2010. Single plasmids expressing human steroid hormone receptors and a reporter gene for use in yeast signaling assays. Plasmid 63: 73-78.

32. Nilsson S, Koehler KF, Gustafsson JA. 2011. Development of subtype-selective oestrogen receptor-based therapeutics. Nat. Rev. Drug Discov. 10: 778-792.

33. Oldenhave A, Jaszmann LJ, Haspels AA, Everaerd WTA. 1993. Impact of climacteric on well-being: a survey based on 5213 women 39 to 60 years old. Am. J. Obstet. Gynecol. 168: 772-780.
34. Paruthiyil S, Cvoro A, Zhao X, Wu Z, Sui Y, Staub RE, et al. 2009. Drug and cell type-specific regulation of genes with different classes of estrogen receptor $\beta$-selective agonists. PLoS One 4: e6271.

35. Pisareva E, Savov V, Kujumdzieva A. 2005. Pigments and citrinin biosynthesis by fungi belonging to genus Monascus. Z. Naturforsch. C J. Biosci. 60: 116-120.

36. Roepke TA, Ronnekleiv OK, Kelly MJ. 2011. Physiological consequences of membrane-initiated estrogen signaling in the brain. Front. Biosci. 16: 1560-1573.

37. Sato Y, He J-X, Nagai H, Tani T, Akao T. 2007. Isoliquiritigenin, one of the antispasmodic principles of Glycyrrhiza ularensis roots, acts in the lower part of intestine. Biol. Pharm. Bull. 30: 145-149.

38. Simmler C, Hajirahimkhan A, Lankin DC, Bolton JL, Jones T, Soejarto DD, et al. 2013. Dynamic residual complexity of the isoliquiritigenin-liquiritigenin interconversion during bioassay. J. Agric. Food Chem. 61: 2146-2157.

39. Song J, Luo J, Ma Z, Sun Q, Wu C-H, Li X. 2019. Quality and authenticity conttrol of functional red yeast rice- a review. Molecules 24(10). pii: E19.

40. Song W, Qiao X, Chen K, Wang Y, Ji S, Feng J, et al. 2017. Biosynthesis-based quantitative analysis of 151 secondary metabolites of licorice to differentiate medicinal Glycyrrhiza species and their hybrids. Anal. Chem. 89: 3146-3153.

41. Su Y-C, Wang J-J, Lin T-T, Pan T-M. 2003. Production of the secondary metabolites $\gamma$-aminobutyric acid and monacolin $\mathrm{K}$ by Monascus. J. Ind. Microbiol. Biotechnol. 30: 41-46.

42. Tamir S, Eizenberg M, Somjen D, Izrael S, Vaya J. 2001. Estrogen-like activity of glabrene and other constituents isolated from licorice root. J. Steroid Biochem. Mol. Biol. 78: 291-298.

43. van Patten CL, Olivotto IA, Chambers GK, Gelmon KA, Hislop TG, Templeton E, et al. 2002. Effect of soy phytoestrogens on hot flashes in postmenopausal women with breast cancer: a randomized, controlled clinical trial. J. Clin. Oncol. 20: 1449-1455.

44. Wang ZY, Nixon DW. 2001. Licorice and cancer. Nutr. Cancer. 39: 1-11.

45. Zhang W, Jiang S, Qian D, Shang Ex, Duan Ja. 2014. Effect of liquiritin on human intestinal bacteria growth: metabolism and modulation. Biomed. Chromatogr. 28: 1271-1277. 